\title{
The Changes of Psychometric Profiles after Medical Treatment of Lower Urinary Tract Symptoms Suggestive of Benign Prostatic Hyperplasia
}

\author{
Kang Jun Cho', Nam Suk Lee ${ }^{1}$, Yong Seok Lee ${ }^{1}$, Woon Jin Jeong ${ }^{2}$, Hong Jin Suh, Joon Chul Kim, Jun Sung Koh ${ }^{1}$ \\ ${ }^{1}$ Department of Urology, College of Medicine, The Catholic University of Korea, ${ }^{2}$ Department of Psychiatry, Seoul Metropolitan Eunpyeong \\ Hospital, Seoul, Korea
}

\begin{abstract}
Objective: To investigate the relationship of somatization and depression with the degree of lower urinary tract symptoms suggestive of benign prostate hyperplasia (LUTS/BPH) and changes in psychometric profiles including somatization and depression after treatment of LUTS/BPH.

Methods: Subjects were evaluated at baseline and at week 12 following routine treatment for LUTS/BPH using the International Prostate Symptom Score (IPSS) to measure the severity of LUTS/BPH, the Overactive Bladder Symptom Score (OABSS) to measure the severity of $\mathrm{OAB}$, the Patient Health Questionnaire-9 (PHQ-9) to assess depression, and the Patient Health Questionnaire-15 (PHQ-15) to evaluate somatization. The correlation of somatization and depression with the degree of LUTS/BPH symptoms at baseline and changes in somatization and depression after LUTS/BPH treatment were assessed using relevant statistical analyses.

Results: One hundred and twenty patients agreed to participate in this study, and 101 (84.2\%) completed the 12-week trial and responded to the study questionnaires. At baseline, total IPSS score was correlated with $\mathrm{PHQ}-9(r=0.475, p=0.005)$ and $\mathrm{PHQ}-15$ $(r=0.596, p<0.001)$ scores. The results after the 12 -week treatment clearly show significant improvement in both $\mathrm{PHQ}-9(p$ $<0.001)$ and $\mathrm{PHQ}-15(p=0.019)$ scores, and the PHQ $-9(r=0.509, p=0.048)$ and $\mathrm{PHQ}-15(r=0.541, p=0.016)$ scores were positively correlated with total IPSS.

Conclusion: Our preliminary results indicated that severity of LUTS is correlated with severity of somatization and depression. Further, the improvement of LUTS after treatment may have positive impacts on somatization and depression.
\end{abstract}

KEY WORDS: Prostatic hyperplasia; Depression; Lower urinary tract symptoms; Somatization disorders; Treatment outcome.

\section{INTRODUCTION}

Lower urinary tract symptoms (LUTS) are common clinical condition, observed in more than $60 \%$ of both men and women $\geq 40$ years of age. ${ }^{1)}$ The current understanding on the LUTS is that it should not be a confirmative formal diagnosis, but rather a subjective indicator of a disease. Although LUTS may not be considered a life-threatening condition, it has a significant negative impact on the quality of life (QoL) of affected individuals, and QoL decreases as LUTS severity increases.

According to a recent huge cross-sectional population-based study, the negative effects of LUTS were

\footnotetext{
Received: March 30, 2015 / Revised: April 23, 2015

Accepted: April 24, 2015

Address for correspondence: Jun Sung Koh, MD, PhD

Department of Urology, The Catholic University of Korea, Bucheon St. Mary's Hospital, 327 Sosa-ro, Wonmi-gu, Bucheon 14647, Korea Tel: +82-32-340-7070, Fax: +82-32-340-2124 E-mail: gostraight@catholic.ac.kr
}

prominent across several domains of QoL and on overall perceptions of general health status and mental health; $35.9 \%$ of men and $53.3 \%$ of women showed clinical anxiety, while $29.8 \%$ of men and $37.6 \%$ of women had clinical depression in multiple storage, voiding and postmicturition LUTS subgroups. ${ }^{2}$ Moderate LUTS has a similar impact on physical health-related QoL to that of diabetes, high blood pressure, and cancer, whereas the impact of severe LUTS is similar to the effect of a heart attack or stroke. ${ }^{3)}$

Poor health-related QoL and life dissatisfaction can have adverse effects on psychological health. Thus, psychological symptoms may coexist in patients with LUTS. Most studies researching the relationship between LUTS and psychological health have focused on depression or anxiety. LUTS increases the risk of developing a depressive disorder in healthy men. Wong et al. ${ }^{4)}$ showed that moderate-to-severe LUTS was associated with a significantly increased risk of clinically relevant depressive

(c) This is an Open-Access article distributed under the terms of the Creative Commons Attribution Non-Commercial License (http://creativecommons.org/licenses/by-nc/4.0) which permits unrestricted non-commercial use, distribution, and reproduction in any medium, provided the original work is properly cited. 
symptoms in elderly men. Some studies have suggested that overactive bladder (OAB) symptoms, such as nocturia and urinary incontinence, can increase anxiety and depression. ${ }^{5-7)}$ In a multicenter study to assess the impact of LUTS on mental health, men with mixed urinary incontinence had high prevalence rates of anxiety and depression. ${ }^{8)}$

Meanwhile, somatization, which is a tendency to experience and communicate psychological distress in the form of somatic expression and to look for medical help, is also associated with urinary symptoms. ${ }^{9)}$ However, there is currently a lack of research about the relationships between somatization and LUTS and between changes in psychometric symptoms and changes in LUTS.

One of the aims of this study is to investigate the relevance of somatization and depression with the degree of LUTS/benign prostate hyperplasia (BPH) symptoms. In addition, we investigated changes of the somatization symptoms and depression associated with improvement of LUTS.

\section{METHODS}

\section{Study Design}

This study was a 12-week prospective observational study in a naturalistic treatment setting.

\section{Patients}

Men with LUTS/BPH were consecutively recruited at our institution. The recruitment period was from March 2011 to February 2012.

The principal inclusion criteria were men aged $\geq 40$ years and clinical diagnosis of LUTS/BPH evaluated by medical history, careful physical examination (including digital rectal examination) and laboratory tests such as level of prostate specific antigen (PSA). Few exclusion criteria were applied because the study aimed to employ a naturalistic observational research approach. However, patients were excluded from the study who showed: (1) PSA level $>10 \mathrm{ng} / \mathrm{ml}$, (2) history or evidence of prostate cancer by prostate biopsy, (3) previous prostatic surgery, (4) any causes presenting LUTS other than BPH (i.e., neurogenic bladder, bladder neck contracture, urethral stricture, bladder malignancy, acute or chronic prostatitis, or acute or chronic urinary tract infections), or (5) speech or language deficit and cognitive dysfunction.

An alpha-blocker and a 5-alpha-reductase inhibitor (5ARI) were the primary medications utilized for the treatment of LUTS/BPH during the course of this study. Throughout the study period, patients remained on the same medication and the same dosage as at the time of enrollment, with the exception of those who required dosage adjustment for response to treatment or adverse event issues.

The present study followed the Declaration of Helsinki and ethical principles regarding human experimentation and the study protocol was approved by the institutional review board of the Catholic University of Korea, Bucheon St. Mary’s Hospital (HC11OISE0004).

\section{Clinical Outcomes}

\section{Study visit}

All patients were assessed twice, at baseline (week 0) and week 12 . We decided to have only two study visits to enhance patient compliance and convenience as well as to not interfere with routine practices at the treatment setting, although such lack of mid-term visits meant less-frequent assessments of patients for treatment outcomes. However, interim visits for adjustments of medications were allowed as necessary, after consultation with the treating physicians.

\section{Symptom assessments (rating scales)}

Patients were interviewed using a standardized, structured questionnaire. In this way, we collected information about general characteristics, age, urologic diagnosis, education level and occupation. The questionnaires were distributed twice; (1) on the day of first visit and (2) after the 12 -week treatment with medication.

This study utilized the International Prostate Symptom Score (IPSS) and Overactive Bladder Symptom Score (OABSS) to measure the severity of LUTS/BPH, the Korean version of the Patient Health Questionnaire-9 (PHQ-9) to assess depression, ${ }^{10,11)}$ and the Korean version of the Patient Health Questionnaire-15 (PHQ-15) to evaluate somatization. $^{12,13)}$ The IPSS has been the most widely used, reliable and validated 8-item instrument for screening, rapidly diagnosing, tracking the symptoms, and suggesting management of the symptoms of the disease LUTS/BPH. The IPSS is the sum of the 7 scores with a range of 0-35. Men were defined as having severe LUTS if they scored $\geq 20$ on the IPSS, moderate LUTS if they scored from 8 to 19 , and mild LUTS if they scored $\leq 7$. $^{14)}$ The OABSS is a single-symptom score that employs a self-reported questionnaire to quantify OAB symptoms. A total of 3 points with at least 2 points on question 3 was required for a diagnosis of $\mathrm{OAB}$. Total scores of $\leq 5,6-11$, and $>11$ were considered mild, moderate, and severe, 
respectively. ${ }^{15)}$ The PHQ-9 is a self-rating scale comprising the 9 criteria upon which the diagnosis of major depressive episode is based in Diagnostic and Statistical Manual of Mental Disorders-IV. It is only half the length of many other depression rating scales, and a patient can finish it in about 5 minutes, but it has comparable sensitivity and specificity to the longer instruments. Each item is scored on a scale of 0 to 3 , and then the circled numbers are added to yield a score ranging from 0 to 27; PHQ-9 total scores of $0-4, \geq 5, \geq 10, \geq 15$, and $\geq 20$ indicate that a patients has none-to-minimal, mild, moderate, moderately severe, or severe depression, respectively. The PHQ-9 allows clinicians to simultaneously diagnose depression and measure symptom severity. ${ }^{11)}$ In addition, it has been found to have good reliability and validity in measuring depression treatment outcomes. ${ }^{16)}$ We have chosen it as the primary outcome measure for the evaluation of depression because it facilitates screening and assessment of severity under the time pressures of a routine practice.

The PHQ-15 is a convenient and very brief self-rating somatic symptom scale. Previous studies have found that the PHQ-15 had good internal consistency corresponding to criterion indices of physical dysfunction, disability days, clinical visits, and amount of difficulty that patients attributed to their symptoms. ${ }^{13)}$ The PHQ-15 also has good reliability and validity in measuring somatization treatment outcomes. ${ }^{17)}$ It assesses 15 somatic symptoms or symptom clusters that account for more than $90 \%$ of all physical complaints (excluding upper respiratory tract symptoms) reported by outpatients. Each item is rated on a scale from 0 to 2 , and scores are summed to return a final score ranging from 0 to 30 . The severity of somatic symptoms is subdivided into total scores ranges of minimal (0-4), mild (5-9), moderate (10-14), and severe (15-30). Both PHQs have also been validated continuously in various patient populations in primary-care settings worldwide. ${ }^{18,19)}$

\section{Primary endpoints}

Our primary hypothesis addressed whether the improvement of LUTS impacts mood symptoms, such as somatization and depression, in patients with LUTS/BPH. Hence, the primary endpoint of this study was changes in the IPSS, PHQ-9, and PHQ-15 total scores from baseline to week 12 . We additionally included correlations of changes from baseline to week 12 in total IPSS, IPSS subscales, and OABSS values with changes in PHQ-9 and PHQ-15 scores over this period.

\section{Statistical Analyses}

Statistical analyses were performed using the Statistical Package for the Social Sciences (SPSS version 16.0 for Windows; SPSS Inc., Chicago, IL, USA). Continuous data are reported as the mean \pm standard deviation. To investigate the correlations between various components of the IPSS and OABSS with PHQ-9 and PHQ-15 scores at baseline, we used a Spearman correlation analysis. To analyze changes in these scores after 12 weeks of treatments, we concluded paired $t$-tests. We also performed Spearman correlation analysis to evaluate correlations of the changes in total IPSS with the changes in total PHQ-9 and PHQ-15 scores. Statistical significance was set at $p$ $<0.05$, two tailed, and there was no adjustment for multiple comparisons because the sample size was relatively small.

\section{RESULTS}

Of the 180 patients screened, 120 agreed to be enrolled in the study and $101(84.2 \%)$ returned for the completion of the treatment as well as completion of questionnaires. Descriptive data of the demographic and clinical characteristics of all participants are summarized in Table 1.

The mean age of the participants was approximately 62 (61.64 \pm 7.81$)$ years and the majority (81.2\%) of subjects were married. The mean baseline IPSS and OABSS total scores were approximately 17 and 5, respectively indicating a moderate severity of LUTS/BPH symptoms. The mean total scores of PHQ-9 and PHQ-15 were each approximately 5 , indicating mild depression and somatization. At baseline, correlation analysis shows that total IPSS correlated with total scores of PHQ-9 $(r=0.475, p=0.005)$ and PHQ-15 $(r=0.596, p<0.001)$.

\section{Primary Endpoints}

The results after the 12-week treatment clearly showed a significant improvement in total scores of IPSS (from $16.53 \pm 8.01$ to $12.02 \pm 7.14, p<0.001$ ), OABSS (from $5.19 \pm$ 3.04 to $3.95 \pm 2.45, p<0.001$ ), PHQ-9 (from $4.58 \pm 4.36$ to $3.46 \pm 4.09, p<0.001$ ) and PHQ-15 (from $4.90 \pm 4.21$ to $4.30 \pm 4.05, p=0.019$ ), as shown in Table 2 .

Finally, correlation analysis showed that the change in IPSS total scores was significantly correlated with the change in PHQ-9 $(r=0.509, p=0.048)$ and PHQ-15 $(r=0.541$, $p=0.016$ ) total scores (Table 3 ). 
Table 1. Patients' demographics and baseline characteristics

\begin{tabular}{|c|c|}
\hline Characteristic & Patient $(n=101)$ \\
\hline Mean age $(y r)$ & $61.64 \pm 7.81$ \\
\hline Duration of disease (mo) & $13.61 \pm 14.85$ \\
\hline \multicolumn{2}{|l|}{ Education level } \\
\hline College or above & $39(38.6)$ \\
\hline Below & $62(61.4)$ \\
\hline \multicolumn{2}{|l|}{ Marital status } \\
\hline Married & $82(81.2)$ \\
\hline Not married & $19(18.8)$ \\
\hline \multicolumn{2}{|l|}{ Work status } \\
\hline Working & $65(64.4)$ \\
\hline Not working & $36(35.6)$ \\
\hline \multicolumn{2}{|l|}{ Medical history } \\
\hline Hypertension & $41(40.6)$ \\
\hline Diabetes mellitus & $31(30.7)$ \\
\hline Hyperlipidemia & $22(21.8)$ \\
\hline IPSS total score & $16.53 \pm 8.01$ \\
\hline Mild (0-7) & $12(11.9)$ \\
\hline Moderate (8-19) & $54(53.5)$ \\
\hline Severe (20-35) & $35(34.6)$ \\
\hline OABSS total score & $5.19 \pm 3.04$ \\
\hline Mild (0-5) & $61(60.4)$ \\
\hline Moderate (6-11) & $37(36.6)$ \\
\hline Severe $(>11)$ & $3(3.0)$ \\
\hline PHQ-9 total score & $4.58 \pm 4.36$ \\
\hline Normal to minimal depression (0-4 points) & $63(62.4)$ \\
\hline Mild depression (5-9) & $21(20.8)$ \\
\hline Moderate depression (10-14) & $14(13.8)$ \\
\hline Moderately severe depression (15-19) & $3(3.0)$ \\
\hline Severe depression (20-27) & $0(0)$ \\
\hline PHQ-15 total score & $4.90 \pm 4.21$ \\
\hline Minimal somatic symptom severity (0-4 points) & $53(52.5)$ \\
\hline Low somatic symptom severity (5-9) & $37(36.6)$ \\
\hline Medium somatic symptom severity (10-14) & $8(7.9)$ \\
\hline High somatic symptom severity ( 15 or more) & $3(3.0)$ \\
\hline
\end{tabular}

Values are presented as mean \pm standard deviation or standard error or number (\%).

IPSS, International Prostate Symptom Score; OABSS, Overactive Bladder Symptom Score; $\mathrm{PHQ}$. Physical Health Questionnaire.

\section{DISCUSSION}

Although there have been several reports suggesting that LUTS are related with mental health problems such as depression, a dearth of information exists for the responses of psychometric manifestations after treatment of LUTS. This preliminary study shows that the total IPSS at baseline were correlated with the psychometric profiles evaluating depression and somatization, and the changes of psychometric profiles with respect to depression and somatization were correlated with the improvements in IPSS after 12 weeks of treatment for LUTS/BPH with an alpha-blocker or 5ARI.

The results of this study showing the relevance of psychological manifestations to the degree of LUTS are similar to those of previous studies. In a large prospective
Table 2. The changes in clinical outcomes in the study $(n=101)$

\begin{tabular}{lcrr}
\hline \multicolumn{1}{c}{ Variable } & At baseline & Week 12 & p value* \\
\hline IPSS total & $16.53 \pm 8.01$ & $12.02 \pm 7.14$ & $<0.001$ \\
IPSS obstruction & $10.04 \pm 5.39$ & $7.11 \pm 4.83$ & $<0.001$ \\
IPSS storage & $6.49 \pm 3.67$ & $4.91 \pm 3.21$ & $<0.001$ \\
Quality of life & $3.46 \pm 1.47$ & $2.51 \pm 1.51$ & $<0.001$ \\
OABSS & $5.19 \pm 3.04$ & $3.95 \pm 2.45$ & $<0.001$ \\
PHQ-9 & $4.58 \pm 4.36$ & $3.46 \pm 4.09$ & $<0.001$ \\
PHQ-15 & $4.90 \pm 4.21$ & $4.30 \pm 4.05$ & 0.019 \\
\hline
\end{tabular}

Values are presented as mean \pm standard deviation.

IPSS, International Prostate Symptom Score; OABSS, Overactive Bladder Symptom Score; $\mathrm{PHQ}$. Physical Health Questionnaire.

*Analysis of paired t-test.

Table 3. The correlation analysis between the mean changes in IPSS and PHQ-9, PHQ-15 scores after treatment $(n=101)$

\begin{tabular}{lcc}
\hline & IPSS total \& $\mathrm{PHQ}-9$ & IPSS total \& $\mathrm{PHQ}-15$ \\
\hline$r$ & 0.509 & 0.541 \\
$p$ value $^{*}$ & 0.048 & 0.016 \\
\hline
\end{tabular}

IPSS, International Prostate Symptom Score; PHQ, Physical Health Questionnaire; $r$, correlation coefficient.

*Spearman correlation analysis.

study of Southern Chinese men, the presence of moderate-to-severe LUTS, measured by the IPSS, at baseline were significantly associated with increased risk of depression at two-year follow-up with adjustments for other possible influencing factors such as demographic characteristics and medical conditions. ${ }^{20)}$ However, we identified that not only depression but also somatization is related with LUTS. To our knowledge, this is the first study to demonstrate a relationship between somatization and LUTS. Somatization causes social and economic problems and diagnostic confusion due to medically unexplained physical symptoms, and it is usually often associated with depression and anxiety disorders. ${ }^{21)}$ Somatization can be a contributing factor affecting the nature or severity of LUTS, and in turn, through physical or organic disturbance, LUTS can pose somatization. This study was conducted to assess the latter notion. In other words, patients with LUTS are more likely than those without LUTS to complain of gastrointestinal symptoms, cardiovascular symptoms, respiratory symptoms, pain disorders and fatigue for which the medical cause cannot be ascertained. Stone et al. ${ }^{22)}$ explained the etiology of somatoform conditions, which can be stratified as biological, psychological and social factors. Although the mechanism of somatization by LUTS is not clear, a possible explanation is an abnormal physiological state caused by LUTS as a biological factor and depression associated with LUTS as a psychological factor. 
The preferred medical treatment for LUTS/BPH is an alpha-blocker or a 5ARI, and improvement of IPSS or OABSS using those medical treatments has been reported in several studies. ${ }^{23,24)}$ An alpha-blocker alone or in combination with 5ARI, was used for the treatment of LUTS/BPH in this study, and after 12 weeks of this treatment, IPSS and OABSS were significantly improved. In addition, medical treatment for LUTS/BPH also improved PHQ-9 and PHQ-15 scores, and their improvements were related to the changes in IPSS total scores, IPSS obstruction subscores and IPSS QoL scores. Although OABSS and IPSS both improved significantly with medical treatments, the IPSS storage subscore and OABSS did not show a statistically significant correlation between depression and somatization in this study. The average OABSS total score at baseline was about 5, and more than $60 \%$ of all patients had a mild OABSS total score. As a result, storage symptoms in this study seemed to carry less weight than obstructive symptoms in patient's treatment satisfaction and so might have little effect on mental health.

Some evidences of an association between improved LUTS and mental health has been shown. Quek et al. ${ }^{25,26)}$ showed that treatment for LUTS, especially surgical treatment, could improve depression, anxiety, and psychiatric morbidity. They suggested the reduction of psychiatric morbidity could be affected by the improvement of LUTS, pain, bother and QoL. In study about the impact of treatment of stress urinary incontinence on mood symptoms, surgical treatment, which have higher success rate than conservative treatment, significantly improved anxiety, depression and QoL compared to the pelvic floor training. ${ }^{27)}$ These results suggest that improvement of mental health may associate with QoL. The correlation of mental disorder with QoL has been previously reported in several other diseases, such as diabetes ${ }^{28)}$ and cardiovascular diseases, ${ }^{29)}$ and even in the general population. ${ }^{30)}$ A correlation between change of IPSS QoL score and changes of depression and somatization was shown in this study. Although IPSS may not be sufficient to measure QoL because it has only one domain of symptom-specific QoL, it has been recommended that the assessment of QoL in patients with BPH be performed with instruments specific to that disease. Thus this study supports the association between mental disorder and QoL in LUTS. However, it is unclear whether improvements in QoL are a result of relief of mental disorders or a possible cause of improvements in depression and somatization.

This study had some limitations. First, we could not an- alyze the objective data such as uroflowmetry and prostate ultrasonography; we instead used self-reports to assess changes in symptoms. However, the symptom questionnaires have been used to evaluate LUTS in clinical practice and can affect the choice of treatment for LUTS. Second, the significance of improvement of PHQ-9 and PHQ-15 total score after treatment should be interpreted with caution, because the magnitude of improvement in each rating scale was small from 4.58 to 3.46 and from 4.90 to 4.30 respectively. Although the improvements were statistically significant, the clinical relevance could be limited, as there is no evidence to suggest substantial improvement in clinical symptoms with slight changes of questionnaire scores. Third, our study had a relatively small sample size that did not have statistical power sufficient to show treatment response and symptom severity in patients with LUTS/BPH. In addition, the observation period in the current study was only 12 weeks, which may not be long enough to fully evaluate the relationship between mental disorder and treatment response. However, there is no current consensus on confirmatively adequate duration of treatment for LUTS/BPH. Finally we did not include control group to compare with the LUTS/BPH group.

Our preliminary results indicated that severity of LUTS is correlated with severity of somatization and depression. In addition, an improvement of LUTS after treatment may have positive impacts on mood symptoms, such as somatization and depression. Thus, it appears that active and early management of LUTS is necessary for mental health and QoL. Further prospective research with adequate power and improved design are needed to provide more information about the relationship of LUTS with mental health.

\section{- Acknowledgments}

This study was funded by the Astellas Korea. The authors have nothing to disclose.

\section{REFERENCES}

1. Irwin DE, Milsom I, Hunskaar S, Reilly K, Kopp Z, Herschorn S, et al. Population-based survey of urinary incontinence, overactive bladder, and other lower urinary tract symptoms in five countries: results of the EPIC study. Eur Urol 2006;50:1306-1314; discussion 1314-1315.

2. Coyne KS, Wein AJ, Tubaro A, Sexton CC, Thompson CL, Kopp ZS, et al. The burden of lower urinary tract symptoms: evaluating the effect of LUTS on health-related quality of life, anxiety and depression: EpiLUTS. BJU Int 2009;103 Suppl 3:4-11.

3. Robertson C, Link CL, Onel E, Mazzetta C, Keech M, Hobbs R, et al. The impact of lower urinary tract symptoms and comorbidities on quality of life: the BACH and UREPIK 
studies. BJU Int 2007;99:347-354.

4. Wong SY, Hong A, Leung J, Kwok T, Leung PC, Woo J. Lower urinary tract symptoms and depressive symptoms in elderly men. J Affect Disord 2006;96:83-88.

5. Yoo ES, Kim BS, Kim DY, Oh SJ, Kim JC. The impact of overactive bladder on health-related quality of life, sexual life and psychological health in Korea. Int Neurourol J 2011;15:143-151.

6. Breyer BN, Shindel AW, Erickson BA, Blaschko SD, Steers $\mathrm{WD}$, Rosen RC. The association of depression, anxiety and nocturia: a systematic review. J Urol 2013;190:953-957.

7. Felde G, Bjelland I, Hunskaar S. Anxiety and depression associated with incontinence in middle-aged women: a large Norwegian cross-sectional study. Int Urogynecol J 2012;23: 299-306.

8. Coyne KS, Kvasz M, Ireland AM, Milsom I, Kopp ZS, Chapple CR. Urinary incontinence and its relationship to mental health and health-related quality of life in men and women in Sweden, the United Kingdom, and the United States. Eur Urol 2012;61:88-95.

9. Lai HH, North CS, Andriole GL, Sayuk GS, Hong BA. Polysymptomatic, polysyndromic presentation of patients with urological chronic pelvic pain syndrome. J Urol 2012;187:2106-2112.

10. Han C, Jo SA, Kwak JH, Pae CU, Steffens D, Jo I, et al. Validation of the Patient Health Questionnaire-9 Korean version in the elderly population: the Ansan Geriatric study. Compr Psychiatry 2008;49:218-223.

11. Kroenke K, Spitzer RL, Williams JB. The PHQ-9: validity of a brief depression severity measure. $J$ Gen Intern Med 2001;16:606-613.

12. Han C, Pae CU, Patkar AA, Masand PS, Kim KW, Joe SH, et al. Psychometric properties of the Patient Health Questionnaire-15 (PHQ-15) for measuring the somatic symptoms of psychiatric outpatients. Psychosomatics 2009; 50:580-585.

13. Kroenke K, Spitzer RL, Williams JB. The PHQ-15: validity of a new measure for evaluating the severity of somatic symptoms. Psychosom Med 2002;64:258-266.

14. Barry MJ, Fowler FJ Jr, O'Leary MP, Bruskewitz RC, Holtgrewe HL, Mebust WK, et al. The American Urological Association symptom index for benign prostatic hyperplasia. The Measurement Committee of the American Urological Association. J Urol 1992;148:1549-1557; discussion 1564.

15. Homma Y, Yoshida M, Seki N, Yokoyama O, Kakizaki H, Gotoh M, et al. Symptom assessment tool for overactive bladder syndrome--overactive bladder symptom score. Urology 2006;68:318-323.

16. Löwe B, Unützer J, Callahan CM, Perkins AJ, Kroenke K. Monitoring depression treatment outcomes with the patient health questionnaire-9. Med Care 2004;42:1194-1201.

17. Han C, Pae CU, Lee BH, Ko YH, Masand PS, Patkar AA, et al. Venlafaxine versus mirtazapine in the treatment of undifferentiated somatoform disorder: a 12-week prospective, open-label, randomized, parallel-group trial. Clin Drug Investig 2008;28:251-261.

18. Moore M, Ali S, Stuart B, Leydon GM, Ovens J, Goodall $\mathrm{C}$, et al. Depression management in primary care: an observational study of management changes related to PHQ-9 score for depression monitoring. Br J Gen Pract 2012;62:e451-e457.

19. Interian A, Allen LA, Gara MA, Escobar JI, Díaz-Martínez AM. Somatic complaints in primary care: further examining the validity of the Patient Health Questionnaire (PHQ-15). Psychosomatics 2006;47:392-398.

20. Chung RY, Leung JC, Chan DC, Woo J, Wong CK, Wong SY. Lower urinary tract symptoms (LUTS) as a risk factor for depressive symptoms in elderly men: results from a large prospective study in Southern Chinese men. PLoS One 2013;8:e76017.

21. Lipowski ZJ. Somatization: the concept and its clinical application. Am J Psychiatry 1988;145:1358-1368.

22. Stone J, Carson A, Sharpe M. Functional symptoms in neurology: management. J Neurol Neurosurg Psychiatry 2005;76 Suppl 1:i13-i21.

23. Roehrborn CG, Siami P, Barkin J, Damião R, Major-Walker $\mathrm{K}$, Nandy I, et al; CombAT Study Group. The effects of combination therapy with dutasteride and tamsulosin on clinical outcomes in men with symptomatic benign prostatic hyperplasia: 4-year results from the CombAT study. Eur Urol 2010;57:123-131.

24. McConnell JD, Roehrborn CG, Bautista OM, Andriole GL $\mathrm{Jr}$, Dixon CM, Kusek JW, et al; Medical Therapy of Prostatic Symptoms (MTOPS) Research Group. The long-term effect of doxazosin, finasteride, and combination therapy on the clinical progression of benign prostatic hyperplasia. N Engl J Med 2003;349:2387-2398.

25. Quek KF, Razack AH, Chua CB, Low WY, Loh CS. Effect of treating lower urinary tract symptoms on anxiety, depression and psychiatric morbidity: a one-year study. Int $J$ Urol 2004;11:848-855.

26. Quek KF, Low WY, Razack AH, Loh CS. The psychological effects of treatments for lower urinary tract symptoms. BJU Int 2000;86:630-633.

27. Innerkofler $P C$, Guenther $V$, Rehder $P$, Kopp $M$, Nguyen-Van-Tam DP, Giesinger JM, et al. Improvement of quality of life, anxiety and depression after surgery in patients with stress urinary incontinence: results of a longitudinal short-term follow-up. Health Qual Life Outcomes 2008;6:72.

28. Nicolau J, Rivera R, Francés C, Chacártegui B, Masmiquel L. Treatment of depression in type 2 diabetic patients: effects on depressive symptoms, quality of life and metabolic control. Diabetes Res Clin Pract 2013;101:148-152.

29. Serafini G, Pompili M, Innamorati M, Iacorossi G, Cuomo I, Della Vista M, et al. The impact of anxiety, depression, and suicidality on quality of life and functional status of patients with congestive heart failure and hypertension: an observational cross-sectional study. Prim Care Companion $J$ Clin Psychiatry 2010;12:PCC.09m00916.

30. Strine TW, Kroenke K, Dhingra S, Balluz LS, Gonzalez O, Berry JT, et al. The associations between depression, health-related quality of life, social support, life satisfaction, and disability in community-dwelling US adults. J Nerv Ment Dis 2009;197:61-64. 